\title{
ASYMPTOTICS OF MAXIMA OF STRONGLY DEPENDENT GAUSSIAN PROCESSES
}

\author{
ZHONGQUAN TAN, ${ }^{*}$ Soochow University \\ ENKELEJD HASHORVA, ${ }^{* *}$ University of Lausanne \\ ZUOXIANG PENG, ${ }^{* * *}$ Southwest University
}

\begin{abstract}
Let $\left\{X_{n}(t), t \in[0, \infty)\right\}, n \in \mathbb{N}$, be standard stationary Gaussian processes. The limit distribution of $\sup _{t \in[0, T(n)]}\left|X_{n}(t)\right|$ is established as $r_{n}(t)$, the correlation function of $\left\{X_{n}(t), t \in[0, \infty)\right\}, n \in \mathbb{N}$, which satisfies the local and long-range strong dependence conditions, extending the results obtained in Seleznjev (1991).

Keywords: Stationary Gaussian process; strong dependence; Berman's condition; limit theorem; Pickands' constant

2010 Mathematics Subject Classification: Primary 60G15

Secondary $60 \mathrm{G} 70$
\end{abstract}

\section{Introduction}

Let $\{X(t), t \in[0, \infty)\}$ be a standard (mean-zero and unit-variance) stationary Gaussian process with continuous sample paths, and let $\{r(t), t \geq 0\}$ denote its correlation function. Assume that the correlation function $r(t)$ of the process satisfies

$$
r(t)=1-|t|^{\alpha}+o\left(|t|^{\alpha}\right) \quad \text { as } t \rightarrow 0 \quad \text { and } \quad r(t)<1 \quad \text { for } t>0
$$

for some $\alpha \in(0,2]$, and further assume that

$$
r(t) \log t \rightarrow 0 \quad \text { as } t \rightarrow \infty
$$

For the study of the asymptotic properties of the supremum of Gaussian processes, the local condition (1.1) is standard, whereas condition (1.2) is the weak dependence condition, or the so-called Berman condition; see, e.g. Piterbarg (1996). Under these two conditions on the correlation function $r(t)$, it is well known (see, e.g. Leadbetter et al. (1983, p. 237) or Berman (1992, p. 212)) that

$$
\lim _{T \rightarrow \infty} \sup _{x \in \mathbb{R}}\left|\mathrm{P}\left\{a_{T}\left(\sup _{t \in[0, T]} X(t)-b_{T}\right) \leq x\right\}-\exp \left(-\mathrm{e}^{-x}\right)\right|=0,
$$

where

$$
a_{T}=\sqrt{2 \log T}, \quad b_{T}=\sqrt{2 \log T}+\frac{\log \left(\mathscr{H}_{\alpha}(2 \pi)^{-1 / 2}(2 \log T)^{-1 / 2+1 / \alpha}\right)}{\sqrt{2 \log T}} .
$$

Received 13 September 2011; revision received 29 March 2012.

* Current address: College of Mathematics, Physics and Information Engineering, Jiaxing University, Jiaxing 314001, P. R. China.

** Postal address: Department of Actuarial Science, Faculty of Business and Economics, University of Lausanne, UNIL-Dorigny, 1015 Lausanne, Switzerland. Email address: enkelejd.hashorva@unil.ch

*** Postal address: School of Mathematics and Statistics, Southwest University, Chongqing 400715, P. R. China. 
Here $\mathscr{H}_{\alpha}$ denotes the Pickands constant defined by $\mathscr{H}_{\alpha}=\lim _{\lambda \rightarrow \infty} \lambda^{-1} \mathscr{H}_{\alpha}(\lambda)$, where

$$
\mathscr{H}_{\alpha}(\lambda)=\mathrm{E}\left\{\exp \left(\max _{t \in[0, \lambda]} \sqrt{2} B_{\alpha / 2}(t)-t^{\alpha}\right)\right\}
$$

and $B_{\alpha}$ is a fractional Brownian motion (a mean-zero Gaussian process with stationary increments such that $\left.\mathrm{E}\left\{B_{\alpha}^{2}(t)\right\}=|t|^{2 \alpha}, t \in \mathbb{R}\right)$. It is also well known that $0<\mathscr{H}_{\alpha}<\infty$; see, e.g. Berman (1992, p. 206) and Piterbarg (1996, p. 16).

In this paper, the following Pickands exact asymptotics play a crucial role in deriving the limit relation of (1.3). Specifically, for some fixed constant $h>0$,

$$
\mathrm{P}\left\{\sup _{t \in[0, h]} X(t)>u\right\}=h \mu(u)(1+o(1)) \quad \text { as } u \rightarrow \infty,
$$

provided that the correlation function $r(t)$ satisfies (1.1) and

$$
\mu(u)=\mathscr{H}_{\alpha} u^{2 / \alpha} \Psi(u),
$$

where $\Psi(\cdot)$ is the survival function of a standard Gaussian random variable. For more details, see Leadbetter et al. (1983, p. 232) and Piterbarg (1996, p. 16). A correct proof of Pickands' theorem (see Pickands (1969)) was given in Piterbarg (1972); for the main properties of Pickands and related constants, see Adler (1990), Berman (1992), Shao (1996), Dieker (2005), Dȩbicki and Kisowski (2009), and Albin and Choi (2010).

A uniform version of (1.5) for stationary Gaussian processes has been established in Seleznjev (1991), where the author investigated the limit distribution of the error of approximation of Gaussian stationary periodic processes by random trigonometric polynomials in the uniform metric. Next, we formulate the aforementioned result.

Theorem 1.1. Let $\left\{X_{n}(t), t \in[0, \infty)\right\}, n \in \mathbb{N}$, be standard stationary Gaussian processes with almost surely (a.s.) continuous sample paths and correlation function $r_{n}(t)$. Let $T(n)>0$ and $u_{n}, n \geq 1$, be constants such that $\lim _{n \rightarrow \infty} \min \left(T(n), u_{n}\right)=\infty$. Suppose further that

(A1) $r_{n}(t)=1-c_{n}|t|^{\alpha}+\varepsilon_{n}(t)|t|^{\alpha}, 0<\alpha \leq 2$, where $c_{n} \rightarrow 1$ as $n \rightarrow \infty$ and $\varepsilon_{n}(t) \rightarrow 0$ as $t \rightarrow 0$ uniformly in $n$;

(A2) for any $\varepsilon>0$, there exists $\gamma>0$ such that $\sup \left\{\left|r_{n}(t)\right|, T \geq|t| \geq \varepsilon, n \in \mathbb{N}\right\}<\gamma<1$;

(A3) $r_{n}(t) \log (t) \rightarrow 0$ as $t \rightarrow \infty$ uniformly in $n$.

Then the following assertions hold.

(i) If (A1) and (A2) hold, then, for any fixed $h>0$ and $\mu(\cdot)$ defined in (1.6),

$$
\lim _{n \rightarrow \infty} \frac{\mathrm{P}\left\{\sup _{t \in[0, h]}\left|X_{n}(t)\right|>u_{n}\right\}}{2 h \mu\left(u_{n}\right)}=1 .
$$

(ii) If, additionally, $\lim _{n \rightarrow \infty} T(n) \mu\left(u_{n}\right)=\theta \in(0, \infty]$ and (A3) holds, then

$$
\lim _{n \rightarrow \infty} \mathrm{P}\left\{\sup _{t \in[0, T(n)]}\left|X_{n}(t)\right| \leq u_{n}\right\}=\mathrm{e}^{-2 \theta},
$$

where we set $\mathrm{e}^{-2 \theta}=0$ if $\theta=\infty$. 
(iii) If instead of assumptions (A1)-(A3), the correlation functions $r_{n}(t)$ are such that

$$
1-r_{n}(t) \leq|t|^{\alpha}, \quad t \in[0, T(n)],
$$

with $\alpha \in(0,2]$ and $T(n) \geq T_{0}>0$ for all large $n$, then

$$
\lim _{n \rightarrow \infty} \mathrm{P}\left\{\sup _{t \in[0, T(n)]}\left|X_{n}(t)\right| \leq u_{n}\right\}=1,
$$

provided that $\lim _{n \rightarrow \infty} T(n) \mu\left(u_{n}\right)=0$.

(iv) Let $a_{T(n)}$ and $b_{T(n)}$ be defined as in (1.4). If (A1), (A2), and (A3) hold, then

$$
\lim _{n \rightarrow \infty} \sup _{x \in \mathbb{R}}\left|\mathrm{P}\left\{a_{T(n)}\left(\sup _{t \in[0, T(n)]}\left|X_{n}(t)\right|-b_{T(n)}\right) \leq x\right\}-\exp \left(-2 \mathrm{e}^{-x}\right)\right|=0 .
$$

The above result has been extended in Seleznjev (1996) to a certain class of nonstationary Gaussian processes. For further extensions and related studies, we refer the reader to Hüsler (1999), Hüsler et al. (2003), and Seleznjev (2006).

Motivated by Seleznjev (1991), in this paper we present the corresponding version of Theorem 1.1 for a sequence of strongly dependent stationary Gaussian processes (see the definition below).

The paper is organized as follows. In Section 2 we give the main results, and in Section 3 we present the proofs.

\section{Main results}

In this section we extend Theorem 1.1 to a sequence of strongly dependent stationary Gaussian processes. A sequence of standard stationary Gaussian process $\left\{X_{n}(t), t \in[0, \infty)\right\}$, $n \in \mathbb{N}$, is called strongly dependent if the correlation function $r_{n}(t)$ satisfies one of the following assumptions:

(B1) $r_{n}(t) \log t \rightarrow r \in(0, \infty)$ as $t \rightarrow \infty$ uniformly in $n$;

(B2) $r_{n}(t) \log t \rightarrow \infty$ as $t \rightarrow \infty$ uniformly in $n$.

Indeed, assumptions (B1) and (B2) are natural extensions of assumption (A3). For related studies on extremes for strongly dependent Gaussian processes, we refer the reader to Mital and Ylvisaker (1975), Piterbarg (1996), Ho and McCormick (1999), and Stamatovic and Stamatovic (2010).

In the following let $\varphi$ and $\Phi$ denote the probability density function and the distribution function of a standard Gaussian random variable $\mathcal{W}$, respectively, and set

$$
\Lambda_{r}(x)=\mathrm{E}\left\{[\Lambda(x+r)]^{\mathrm{e}^{\sqrt{2 r} w}}+\mathrm{e}^{-\sqrt{2 r} w}\right\}, \quad x \in \mathbb{R},
$$

with $\Lambda(x)=\exp (-\exp (-x)), x \in \mathbb{R}$, the unit Gumbel distribution function.

Next, we state our main results. 
Theorem 2.1. Let $\left\{X_{n}(t), t \in[0, \infty)\right\}, n \in \mathbb{N}$, be standard stationary Gaussian processes with a.s. continuous sample paths and correlation function $r_{n}(t)$ satisfying $(A 1),(A 2)$, and (B1).

(i) If $\lim _{n \rightarrow \infty} T(n) \mu\left(u_{n}\right)=\theta \in(0, \infty]$ then

$$
\lim _{n \rightarrow \infty} \mathrm{P}\left\{\sup _{t \in[0, T(n)]}\left|X_{n}(t)\right| \leq u_{n}\right\}=\Lambda_{r}(-\log \theta),
$$

where $\Lambda_{r}(-\log \theta)=$ : 0 if $\theta=\infty$.

(ii) Let $a_{T(n)}$ and $b_{T(n)}$ be defined as in (1.4). For $x \in \mathbb{R}$, we have

$$
\left.\lim _{n \rightarrow \infty} \sup _{x \in \mathbb{R}} \mid \mathrm{P}\left\{a_{T(n)}\left(\sup _{t \in[0, T(n)]}\left|X_{n}(t)\right|-b_{T(n)}\right) \leq x\right\}-\Lambda_{r}(x)\right\}=0 .
$$

Remark 2.1. (a) From the proof of Theorem 2.1 below, it follows that both (2.1) and (2.2) can be shown to hold for $r=0$ also, thus retrieving the result of Theorem 1.1.

(b) Assertion (iii) of Theorem 1.1 still holds under the conditions of Theorem 2.1.

Theorem 2.2. Let $\left\{X_{n}(t), t \in[0, \infty)\right\}, n \in \mathbb{N}$, be standard stationary Gaussian processes with a.s. continuous sample paths and correlation function $r_{n}(t)$ satisfying $(A 1)$ with $0<\alpha \leq 1$, $(A 2)$, and (B2). Assume that $r_{n}(t)$ is convex for $t \geq 0$ and $r_{n}(t)=o(1)$ uniformly in $n$. If, furthermore, $r_{n}(t) \log t$ is monotone for large $t$ then, with $b_{T(n)}$ as in (1.4), we have

$$
\begin{aligned}
& \lim _{n \rightarrow \infty} \sup _{x \in(0, \infty)} \mid \mathrm{P}\left\{r_{n}^{-1 / 2}(T(n))\left(\sup _{t \in[0, T(n)]}\left|X_{n}(t)\right|-\left(1-r_{n}(T(n))\right)^{1 / 2} b_{T(n)}\right) \leq x\right\} \\
& \quad-2 \Phi(x)+1 \mid \\
& \quad=0 .
\end{aligned}
$$

Remark 2.2. Theorem 2.2 is a uniform version of Theorem 3.1 of Mittal and Ylvisaker (1975).

\section{Further results and proofs}

We begin with some auxiliary lemmas needed for the proofs of Theorems 2.1 and 2.2.

For given $\varepsilon>0$, we divide the interval $[0, T(n)]$ into intervals of length 1 , and split each of the intervals into the subintervals $I_{j}^{\varepsilon}$ and $I_{j}, j=1,2, \ldots,[T(n)]$, of length $\varepsilon$ and $1-\varepsilon$, respectively, where $[x]$ denotes the integral part of $x$. It can be easily seen that a possible remaining interval with length smaller than 1 plays no role in our consideration. We denote this interval by $J$.

Let $\left\{X_{n}^{(i)}(t), t \geq 0\right\}, i=1,2, \ldots$, be independent copies of $\left\{X_{n}(t), t \geq 0\right\}$, and let $\left\{\eta_{n}(t)\right.$, $t \geq 0\}$ be such that $\eta_{n}(t)=X_{n}^{(j)}(t)$ for $t \in I_{j}$. Let $\rho(T(n)):=r / \log T(n)$, and define

$$
\xi_{n}(t)=(1-\rho(T(n)))^{1 / 2} \eta_{n}(t)+\rho^{1 / 2}(T(n)) W, \quad t \in \bigcup_{j=1}^{[T(n)]} I_{j},
$$

where $\mathcal{W}$ is a standard Gaussian random variable independent of $\left\{\eta_{n}(t), t \geq 0\right\}$. Note that $\left\{\xi_{n}(t), t \in \bigcup_{j=1}^{[T(n)]} I_{j}\right\}$ is a standard Gaussian process with correlation function $\varrho_{n}(\cdot)$, given by

$$
\varrho_{n}(t-s)= \begin{cases}r_{n}(t-s)+\left(1-r_{n}(t-s)\right) \rho(T(n)), & t \in I_{j}, s \in I_{i}, i=j, \\ \rho(T(n)), & t \in I_{j}, s \in I_{i}, i \neq j .\end{cases}
$$


In the sequel, we assume that $a, u_{n}$, and $v_{n}$ are positive constants, and we set

$$
q:=q\left(u_{n}\right)=a u_{n}^{-2 / \alpha}, \quad \mu\left(u_{n}\right):=\mathscr{H}_{\alpha} u_{n}^{2 / \alpha} \Psi\left(u_{n}\right), \quad \delta(a):=1-\frac{\mathscr{H}_{\alpha}(a)}{\mathscr{H}_{\alpha}} .
$$

Furthermore, $C_{1}-C_{6}$ will denote positive constants whose values may vary from place to place.

Lemma 3.1. If assumptions (A1) and (A2) hold then, for each interval I of fixed length $h>0$,

$$
0 \leq \mathrm{P}\left\{\max _{j q \in I}\left|X_{n}(j q)\right| \leq u_{n}\right\}-\mathrm{P}\left\{\sup _{s \in I}\left|X_{n}(s)\right| \leq u_{n}\right\} \leq 2 h \delta(a) \mu\left(u_{n}\right)+o\left(\mu\left(u_{n}\right)\right)
$$

and

$$
0 \leq \mathrm{P}\left\{\max _{j q \in I} X_{n}(j q) \leq u_{n}\right\}-\mathrm{P}\left\{\sup _{s \in I} X_{n}(s) \leq u_{n}\right\} \leq h \delta(a) \mu\left(u_{n}\right)+o\left(\mu\left(u_{n}\right)\right),
$$

where $\delta(a) \rightarrow 0$ as $a \downarrow 0$.

Proof. Both claims above are established in the proof of Theorem 1 of Seleznjev (1991).

Lemma 3.2. Suppose that assumptions (A1) and (A2) hold. If $T(n) \mu\left(u_{n}\right)=O(1)$ and $T(n) \mu\left(v_{n}\right)=O(1)$, then

$$
\mathrm{P}\left\{\sup _{s \in[0, T(n)]}\left|X_{n}(s)\right| \leq u_{n}\right\}-\mathrm{P}\left\{\sup _{s \in \cup I_{j}}\left|X_{n}(s)\right| \leq u_{n}\right\} \rightarrow 0
$$

and

$$
\begin{aligned}
& \mathrm{P}\left\{-v_{n} \leq \inf _{s \in[0,1]} X_{n}(s), \sup _{s \in[0,1]} X_{n}(s) \leq u_{n}\right\}-\mathrm{P}\left\{-v_{n} \leq \inf _{s \in I_{1}} X_{n}(s), \sup _{s \in I_{1}} X_{n}(s) \leq u_{n}\right\} \\
& \rightarrow 0
\end{aligned}
$$

as $n \rightarrow \infty$ and $\varepsilon \downarrow 0$.

Proof. By the stationarity of $\left\{X_{n}(t), t \in[0, T(n)]\right\}$ and Theorem 1.1(i), we obtain

$$
\begin{aligned}
& \left|\mathrm{P}\left\{\sup _{s \in[0, T(n)]}\left|X_{n}(s)\right| \leq u_{n}\right\}-\mathrm{P}\left\{\sup _{s \in \cup I_{j}}\left|X_{n}(s)\right| \leq u_{n}\right\}\right| \\
& \quad \leq \sum_{j=1}^{[T(n)]} \mathrm{P}\left\{\max _{s \in I_{j}^{\varepsilon}}\left|X_{n}(s)\right|>u_{n}\right\}+\mathrm{P}\left\{\max _{s \in J}\left|X_{n}(s)\right|>u_{n}\right\} \\
& \leq 2([T(n)] \varepsilon+1) \mu\left(u_{n}\right)(1+o(1)) \\
& =O(1) \varepsilon(1+o(1)) \\
& \quad \rightarrow 0
\end{aligned}
$$

as $u \rightarrow \infty$ and $\varepsilon \downarrow 0$, which completes the proof of (3.1). We note in passing that

$$
\begin{aligned}
\mid \mathrm{P}\{- & \left.v_{n} \leq \inf _{s \in[0,1]} X_{n}(s), \sup _{s \in[0,1]} X_{n}(s) \leq u_{n}\right\}-\mathrm{P}\left\{-v_{n} \leq \inf _{s \in I_{1}} X_{n}(s), \sup _{s \in I_{1}} X_{n}(s) \leq u_{n}\right\} \mid \\
\leq & \left|\mathrm{P}\left\{\sup _{s \in[0,1]} X_{n}(s) \leq u_{n}\right\}-\mathrm{P}\left\{\sup _{s \in I_{1}} X_{n}(s) \leq u_{n}\right\}\right| \\
& +\left|\mathrm{P}\left\{\inf _{s \in[0,1]} X_{n}(s) \geq-v_{n}\right\}-\mathrm{P}\left\{\inf _{s \in I_{1}} X_{n}(s) \geq-v_{n}\right\}\right| .
\end{aligned}
$$

The proof of (3.2) is similar to that of (3.1), and is therefore omitted. 
Lemma 3.3. Under the assumptions of Lemma 3.2, we have

$$
\mathrm{P}\left\{\sup _{s \in \cup I_{j}}\left|X_{n}(s)\right| \leq u_{n}\right\}-\mathrm{P}\left\{\max _{k q \in \cup I_{j}}\left|X_{n}(k q)\right| \leq u_{n}\right\} \rightarrow 0
$$

and

$$
\begin{aligned}
& \mathrm{P}\left\{-v_{n} \leq \inf _{s \in I_{1}} X_{n}(s), \sup _{s \in I_{1}} X_{n}(s) \leq u_{n}\right\}-\mathrm{P}\left\{-v_{n} \leq \min _{k q \in I_{1}} X_{n}(k q), \max _{k q \in I_{1}} X_{n}(k q) \leq u_{n}\right\} \\
& \rightarrow 0
\end{aligned}
$$

as $n \rightarrow \infty$ and $a \downarrow 0$.

Proof. By Lemma 3.2,

$$
\begin{aligned}
\left|\mathrm{P}\left\{\sup _{s \in \cup I_{j}}\left|X_{n}(s)\right| \leq u_{n}\right\}-\mathrm{P}\left\{\sup _{k q \in \cup I_{j}}\left|X_{n}(k q)\right| \leq u_{n}\right\}\right| \\
\quad \leq T(n) \max _{j}\left(\mathrm{P}\left\{\max _{k q \in I_{j}}\left|X_{n}(k q)\right| \leq u_{n}\right\}-\mathrm{P}\left\{\sup _{s \in I_{j}}\left|X_{n}(s)\right| \leq u_{n}\right\}\right) \\
\quad \leq 2(1-\varepsilon)[T(n)] \mu\left(u_{n}\right) \delta(a)+T(n) o\left(\mu\left(u_{n}\right)\right) \\
\quad=2(1-\varepsilon) O(1) \delta(a)+o(1) \\
\quad \rightarrow 0
\end{aligned}
$$

as $n \rightarrow \infty$ and $a \downarrow 0$. Hence, the first claim follows. Note that

$$
\begin{aligned}
\mid \mathrm{P}\{- & \left.v_{n} \leq \inf _{s \in I_{1}} X_{n}(s), \sup _{s \in I_{1}} X_{n}(s) \leq u_{n}\right\}-\mathrm{P}\left\{-v_{n} \leq \min _{k q \in I_{1}} X_{n}(k q), \max _{k q \in I_{1}} X_{n}(k q) \leq u_{n}\right\} \mid \\
\leq & \left|\mathrm{P}\left\{\max _{k q \in I_{1}} X_{n}(k q) \leq u_{n}\right\}-\mathrm{P}\left\{\sup _{s \in I_{1}} X_{n}(s) \leq u_{n}\right\}\right| \\
& +\left|\mathrm{P}\left\{\min _{k q \in I_{1}} X_{n}(k q) \geq-v_{n}\right\}-\mathrm{P}\left\{\inf _{s \in I_{1}} X_{n}(s) \geq-v_{n}\right\}\right| .
\end{aligned}
$$

We omit the proof of (3.4) since it is similar to that of (3.3).

Lemma 3.4. Suppose that assumptions (A1), (A2), and (B1) hold. If $T(n) \mu\left(u_{n}\right)=O(1)$ then

$$
\lim _{n \rightarrow \infty}\left|\mathrm{P}\left\{\max _{k q \in \cup I_{j}}\left|X_{n}(k q)\right| \leq u_{n}\right\}-\mathrm{P}\left\{\max _{k q \in \cup I_{j}}\left|\xi_{n}(k q)\right| \leq u_{n}\right\}\right|=0 .
$$

Proof. Applying the generalized Berman inequality (cf. Theorem 1.2 of Piterbarg (1996)), we have (setting $T:=T(n)$ )

$$
\begin{aligned}
& \left|\mathrm{P}\left\{\max _{k q \in \cup I_{j}}\left|X_{n}(k q)\right| \leq u_{n}\right\}-\mathrm{P}\left\{\max _{k q \in \cup I_{j}}\left|\xi_{n}(k q)\right| \leq u_{n}\right\}\right| \\
& \leq \sum_{k q \in I_{i}, l q \in I_{j}} \frac{4}{2 \pi}\left|r_{n}(k q, l q)-\varrho_{n}(k q, l q)\right| \\
& \quad \times \int_{0}^{1} \frac{1}{\sqrt{1-r^{(h)}(k q, l q)}} \exp \left(-\frac{u_{n}^{2}}{1+r^{(h)}(k q, l q)}\right) \mathrm{d} h \\
& \leq \sum_{\substack{0<k q, l q \leq T \\
0<|k q-l q| \leq 1-\varepsilon}} \mathbb{A}(n, k, l, q)+\sum_{\substack{0<k q, l q \leq T \\
|k q-l q|>\varepsilon}} \mathbb{A}(n, k, l, q),
\end{aligned}
$$


where $\varphi\left(x, y, r^{(h)}\right)$ is a Gaussian two-dimensional density with covariance $r^{(h)}$, variance equal to 1 , zero mean, and

$$
r^{(h)}(k q, l q)=h r_{n}(k q, l q)+(1-h) \varrho_{n}(k q, l q), \quad h \in[0,1] .
$$

In the following part of the proof, let

$$
\varpi_{n}(k q)=\max \left\{\left|r_{n}(k q)\right|,\left|\varrho_{n}(k q)\right|\right\} \quad \text { and } \quad \vartheta_{n}(t)=\sup _{t<k q \leq T}\left\{\varpi_{n}(k q)\right\} .
$$

By assumption (A2) and the definition of $\varrho_{n}(t)$, we have

$$
\vartheta(\varepsilon)=\sup _{\varepsilon<k q \leq T}\left\{\varpi_{n}(k q) ; n \in \mathbb{N}\right\}<1
$$

for sufficiently large $T$. Furthermore, let $\beta$ be such that $0<\beta<(1-\vartheta(\varepsilon)) /(1+\vartheta(\varepsilon))$ for all sufficiently large $T$.

Next, we estimate the upper bound of (3.6) in the case that $k q$ and $l q$ belong to the same interval $I$. Note that in this case, $\varrho_{n}(k q, l q)=r_{n}(k q-l q)+\left(1-r_{n}(k q-l q)\right) \rho(T) \sim r_{n}(k q-l q)$ for sufficiently large $T$. Split the first term of (3.6) into two parts:

$$
\sum_{\substack{0<k q, l q \leq T \\ 0<|k q-l q| \leq \varepsilon}} \mathbb{A}(n, k, l, q)+\sum_{\substack{0<k q, l q \leq T \\ \varepsilon<|k q-l q| \leq 1-\varepsilon}} \mathbb{A}(n, k, l, q)=: J_{n 1}+J_{n 2} .
$$

Assumption (A1) implies that, for all $|t| \leq \varepsilon<2^{-1 / \alpha}$,

$$
1-r_{n}(t) \leq 2|t|^{\alpha}
$$

From the assumption that $T \mu\left(u_{n}\right)=T(n) \mu\left(u_{n}\right)=O(1)$, we have

$$
u_{n} \sim(2 \log T)^{1 / 2}, \quad \mathrm{e}^{-u_{n}^{2} / 2} \sim(2 \pi)^{1 / 2} H_{\alpha}^{-1} u_{n}^{1-2 / \alpha} T^{-1} O(1) .
$$

Consequently, with $q:=a u_{n}^{-2 / \alpha} \sim a(\log T)^{-1 / \alpha}$ we obtain

$$
\begin{aligned}
J_{n 1} & \leq C_{1} \frac{T}{q} \sum_{0<k q \leq \varepsilon}\left|r_{n}(k q)-\varrho_{n}(k q)\right| \frac{1}{\sqrt{1-\varrho_{n}(k q)}} \exp \left(-\frac{u_{n}^{2}}{1+\varpi_{n}(k q)}\right) \\
& \leq C_{1} \frac{T}{q} \sum_{0<k q \leq \varepsilon}\left|\left(1-r_{n}(k q)\right) \rho(T)\right| \frac{1}{\sqrt{1-r_{n}(k q)}} \exp \left(-\frac{u_{n}^{2}}{2}\right) \\
& \leq C_{1} \frac{T}{q} \rho(T) \exp \left(-\frac{u_{n}^{2}}{2}\right) \sum_{0<k q \leq \varepsilon} \sqrt{1-r_{n}(k q)} \\
& \leq C_{1} \frac{T}{q} \rho(T) T^{-1}(\log T)^{1 / 2-1 / \alpha} \sum_{0<k q \leq \varepsilon} \sqrt{2(k q)^{\alpha}} \\
& \leq C_{1}(\log T)^{-1 / 2},
\end{aligned}
$$


which implies that $\lim _{n \rightarrow \infty} J_{n 1}=0$. By (3.8) for large $T$ we have

$$
\begin{aligned}
J_{n 2} & \leq C_{2} \frac{T}{q} \sum_{\varepsilon<k q \leq 1-\varepsilon}\left|r_{n}(k q)-\varrho_{n}(k q)\right| \exp \left(-\frac{u_{n}^{2}}{1+\varpi_{n}(k q)}\right) \\
& \leq C_{2} \frac{T}{q} \sum_{\varepsilon<k q \leq 1-\varepsilon}\left|r_{n}(k q)-\varrho_{n}(k q)\right| \exp \left(-\frac{u_{n}^{2}}{1+\vartheta(\varepsilon)}\right) \\
& \leq C_{2} \frac{T}{q^{2}}\left(\exp \left(-\frac{u_{n}^{2}}{2}\right)\right)^{(1+\vartheta(\varepsilon)) / 2} \\
& \leq C_{2} T^{-(1-\vartheta(\varepsilon)) /(1+\vartheta(\varepsilon))} u_{n}^{2+(\alpha-2) / \alpha(1+\vartheta(\varepsilon))} \\
& \leq C_{2} T^{-(1-\vartheta(\varepsilon)) /(1+\vartheta(\varepsilon))}(\log T)^{(2|\alpha-1|+\alpha) / \alpha} .
\end{aligned}
$$

Hence, since $\vartheta(\varepsilon)<1, \lim _{n \rightarrow \infty} J_{n 2}=0$.

We continue with an estimate for the upper bound of (3.6) where $k q \in I_{i}$ and $l q \in I_{j}$, $i \neq j$. Note that in this case, the distance between any two intervals $I_{i}$ and $I_{j}$ is larger than $\varepsilon$. Split the second term of (3.6) as

$$
\sum_{\varepsilon<|k q-l q| \leq T^{\beta}} \mathbb{A}(n, k, l, q)+\sum_{T^{\beta}<|k q-l q| \leq T} \mathbb{A}(n, k, l, q)=: I_{n 1}+I_{n 2} .
$$

Similarly to the derivation of (3.10), we have

$$
\begin{aligned}
I_{n 1} & \leq C_{3} \frac{T}{q} \sum_{\varepsilon<k q \leq T^{\beta}}\left|r_{n}(k q)-\varrho_{n}(k q)\right| \exp \left(-\frac{u_{n}^{2}}{1+\varpi_{n}(k q)}\right) \\
& \leq C_{3} \frac{T}{q} \sum_{\varepsilon<k q \leq T^{\beta}}\left|r_{n}(k q)-\varrho_{n}(k q)\right| \exp \left(-\frac{u_{n}^{2}}{1+\vartheta(\varepsilon)}\right) \\
& \leq C_{3} \frac{T^{1+\beta}}{q^{2}}\left(\exp \left(-\frac{u_{n}^{2}}{2}\right)\right)^{(1+\vartheta(\varepsilon)) / 2} \\
& \leq C_{3} T^{\beta-(1-\vartheta(\varepsilon)) /(1+\vartheta(\varepsilon))}(\log T)^{-(2-\alpha+2(1+\vartheta(\varepsilon))) / \alpha(1+\vartheta(\varepsilon))} \\
& \leq C_{3} T^{\beta-(1-\vartheta(\varepsilon)) /(1+\vartheta(\varepsilon))} .
\end{aligned}
$$

Thus, $\lim _{n \rightarrow \infty} I_{n 1}=0$, since $\beta<(1-\vartheta(\varepsilon)) /(1+\vartheta(\varepsilon))$. Furthermore, assumption (B1) implies that there exists a positive constant $K$ such that $\varpi_{n}(k q) \leq K / \log T^{\beta}$ for $k q>T^{\beta}$. Using (3.8) again, for $q=a u_{n}^{-2 / \alpha} \sim a(\log T)^{-1 / \alpha}$, we have

$$
\begin{aligned}
\frac{T^{2}}{q^{2} \log T} \exp \left(-\frac{u_{n}^{2}}{1+\varpi_{n}(k q)}\right) & \leq \frac{T^{2}}{q^{2} \log T} \exp \left(-\frac{u_{n}^{2}}{1+K / \log T^{\beta}}\right) \\
& \leq C_{4} \exp \left(\frac{2 K \log T}{K+\beta \log T}-\left(1-\frac{2}{\alpha}\right) \frac{K \log \log T}{K+\beta \log T}\right) \\
& =O(1) .
\end{aligned}
$$

Hence, following the argument given in the proof of Lemma 6.4.1 of Leadbetter et al. (1983) 
we may further write

$$
\begin{aligned}
I_{n 2} & \leq C_{5} \frac{T}{q} \sum_{T^{\beta}<k q \leq T}\left|r_{n}(k q)-\varrho_{n}(k q)\right| \exp \left(-\frac{u_{n}^{2}}{1+\varpi_{n}(k q)}\right) \\
& =C_{5} \frac{q \log T}{T} \sum_{T^{\beta}<k q \leq T}\left|r_{n}(k q)-\varrho_{n}(k q)\right| \frac{T^{2}}{q^{2} \log T} \exp \left(-\frac{u_{n}^{2}}{1+\varpi_{n}(k q)}\right) \\
& \leq C_{5} \frac{q \log T}{T} \sum_{T^{\beta}<k q \leq T}\left|r_{n}(k q)-\rho(T)\right| \\
& \leq C_{5} \frac{q}{\beta T} \sum_{T^{\beta}<k q \leq T}\left|r_{n}(k q) \log k q-r\right|+C_{6} r \frac{q}{T} \sum_{T^{\beta}<k q \leq T}\left|1-\frac{\log T}{\log k q}\right| .
\end{aligned}
$$

By assumption (B1), the first term on the right-hand side of (3.13) tends to 0. Furthermore, the second term therein also tends to 0 , which follows by an integral estimate, as in the proof of Lemma 6.4.1 of Leadbetter et al. (1983). Consequently, the proof is established by (3.6)-(3.7) and (3.9)-(3.13).

Lemma 3.5. Suppose that assumptions (A1) and (A2) hold. If $T(n) \mu\left(u_{n}\right)=O(1)$ and $T(n) \mu\left(v_{n}\right)=O(1)$, then

$$
\mathrm{P}\left\{\sup _{s \in[0,1]} X_{n}(s)>u_{n}, \inf _{s \in[0,1]} X_{n}(s)<-v_{n}\right\}=o\left(\mu\left(u_{n}\right)+\mu\left(v_{n}\right)\right) \text { as } n \rightarrow \infty .
$$

Proof. The proof is similar to that of Lemma 11.1.4 of Leadbetter et al. (1983).

Proof of Theorem 2.1. We only prove case (i), since case (ii) is a special case of (i).

Case 1: $\theta \in(0, \infty)$. The definition of $\left\{\xi_{n}(t), t \in \bigcup_{j=1}^{[T(n)]} I_{j}\right\}$ implies that

$$
\begin{aligned}
\mathrm{P}\left\{\max _{k q \in \cup I_{j}}\left|\xi_{n}(k q)\right| \leq u_{n}\right\} \\
\quad=\mathrm{P}\left\{\max _{k q \in \cup I_{j}}\left|(1-\rho(T(n)))^{1 / 2} \eta_{n}(k q)+\rho^{1 / 2}(T(n)) \mathcal{W}\right| \leq u_{n}\right\} \\
\quad=\mathrm{P}\left\{-u_{n} \leq(1-\rho(T(n)))^{1 / 2} \eta_{n}(k q)+\rho^{1 / 2}(T(n)) \mathcal{W} \leq u_{n}, k q \in \cup I_{j}\right\} \\
\quad=\int_{-\infty}^{+\infty} \mathrm{P}\left\{\frac{-u_{n}-\rho^{1 / 2}(T(n)) z}{(1-\rho(T(n)))^{1 / 2}} \leq \eta_{n}(k q) \leq \frac{u_{n}-\rho^{1 / 2}(T(n)) z}{(1-\rho(T(n)))^{1 / 2}}, k q \in \cup I_{j}\right\} \varphi(z) \mathrm{d} z .
\end{aligned}
$$

Note that, as $n \rightarrow \infty$,

$$
u_{n}^{(z)}:=\frac{u_{n}-\rho^{1 / 2}(T(n)) z}{(1-\rho(T(n)))^{1 / 2}}=u_{n}+\frac{r-\sqrt{2 r} z}{u_{n}}+o\left(u_{n}^{-1}\right)
$$

and

$$
v_{n}^{(z)}:=\frac{u_{n}+\rho^{1 / 2}(T(n)) z}{(1-\rho(T(n)))^{1 / 2}}=u_{n}+\frac{r+\sqrt{2 r} z}{u_{n}}+o\left(u_{n}^{-1}\right) .
$$

So, the assumption that $\lim _{n \rightarrow \infty} T(n) \mu\left(u_{n}\right)=\theta \in(0, \infty)$ implies that

$$
\lim _{n \rightarrow \infty} T(n) \mu\left(u_{n}^{(z)}\right)=\theta \mathrm{e}^{-r+\sqrt{2 r} z}, \quad \lim _{n \rightarrow \infty} T(n) \mu\left(v_{n}^{(z)}\right)=\theta \mathrm{e}^{-r-\sqrt{2 r} z} .
$$


Next, by the definition of $\left\{\eta_{n}(t), t \geq 0\right\},(3.2),(3.4)$, and (3.15), we have

$$
\begin{aligned}
& \mathrm{P}\left\{-v_{n}^{(z)} \leq \eta_{n}(k q) \leq u_{n}^{(z)}, k q \in \cup I_{j}\right\} \\
&=\prod_{j=1}^{[T(n)]} \mathrm{P}\left\{-v_{n}^{(z)} \leq X_{n}^{(j)}(k q) \leq u_{n}^{(z)}, k q \in I_{j}\right\} \\
&\left.=\mathrm{P}\left\{-v_{n}^{(z)} \leq X_{n}(k q) \leq u_{n}^{(z)}, k q \in I_{1}\right)\right\}^{[T(n)]} \\
&=\mathrm{P}\left\{-v_{n}^{(z)} \leq X_{n}(t) \leq u_{n}^{(z)}, t \in I_{1}\right\}^{[T(n)]}(1+o(1)) \\
&=\mathrm{P}\left\{-v_{n}^{(z)} \leq X_{n}(t) \leq u_{n}^{(z)}, t \in[0,1]\right\}^{[T(n)]}(1+o(1)) \\
&=\left(1-\mathrm{P}\left\{\inf _{s \in[0,1]} X_{n}(s)<-v_{n}^{(z)}\right\}-\mathrm{P}\left\{\sup _{s \in[0,1]} X_{n}(t)>u_{n}^{(z)}\right\}\right. \\
&\left.\quad+\mathrm{P}\left\{\inf _{s \in[0,1]} X_{n}(s)<-v_{n}^{(z)}, \sup _{s \in[0,1]} X_{n}(t)>u_{n}^{(z)}\right\}\right)^{[T(n)]}(1+o(1))
\end{aligned}
$$

as $n \rightarrow \infty$. In light of Theorem 1.1(i) and Lemma 3.5,

$$
\begin{aligned}
\mathrm{P}\{- & \left.v_{n}^{(z)} \leq \eta_{n}(k q) \leq u_{n}^{(z)}, k q \in \cup I_{j}\right\} \\
= & \left(1-\mu\left(u_{n}^{(z)}\right)-\mu\left(v_{n}^{(z)}\right)+o\left(\mu\left(u_{n}^{(z)}\right)+\mu\left(v_{n}^{(z)}\right)\right)\right)^{[T(n)]}(1+o(1)) \\
& =\left(1-\frac{\theta \mathrm{e}^{-(r-\sqrt{2 r} z)}+\theta \mathrm{e}^{-(r+\sqrt{2 r} z)}}{T(n)}+o\left(\frac{1}{T(n)}\right)\right)^{[T(n)]}(1+o(1)) \\
& =\exp \left(-\theta \mathrm{e}^{-(r-\sqrt{2 r} z)}-\theta \mathrm{e}^{-(r+\sqrt{2 r} z)}\right)(1+o(1))
\end{aligned}
$$

as $n \rightarrow \infty$. Combining the last result with (3.5) and (3.14), and applying the dominated convergence theorem, we obtain

$$
\lim _{n \rightarrow \infty} \mathrm{P}\left\{\max _{k q \in \cup I_{j}}\left|X_{n}(k q)\right| \leq u_{n}\right\}=\int_{-\infty}^{+\infty} \exp \left(-\theta \mathrm{e}^{-(r-\sqrt{2 r} z)}-\theta \mathrm{e}^{-(r+\sqrt{2 r} z)}\right) \varphi(z) \mathrm{d} z .
$$

Consequently, the proof follows by further utilising (3.1), (3.3), and (3.5).

Case 2: $\theta=\infty$. From the definition of $\mu(\cdot)$, we know that, for arbitrarily large $\theta^{\prime}<\infty$, there exists a real sequence $v_{n}$ such that $\lim _{n \rightarrow \infty} n \mu\left(v_{n}\right)=\theta^{\prime}$. Clearly, for sufficient large $n$, $u_{n} \leq v_{n}$; hence,

$$
\mathrm{P}\left\{\sup _{t \in[0, T(n)]}\left|X_{n}(t)\right| \leq u_{n}\right\} \leq \mathrm{P}\left\{\sup _{t \in[0, T(n)]}\left|X_{n}(t)\right| \leq v_{n}\right\} \rightarrow \Lambda_{r}\left(-\log \theta^{\prime}\right) \quad \text { as } n \rightarrow \infty .
$$

Since this holds for arbitrarily large $\theta^{\prime}<\infty$, by letting $\theta^{\prime} \rightarrow \infty$ we see that

$$
\lim _{n \rightarrow \infty} \mathrm{P}\left\{\sup _{t \in[0, T(n)]}\left|X_{n}(t)\right| \leq u_{n}\right\}=0,
$$

which completes the proof.

For the proof of Theorem 2.2, we need a result which is formulated in the next lemma. By Polya's criterion (see, e.g. Equation (3.10) of Durrett (2004)), if we assume the convexity of the correlation functions $r_{n}(t)$ (hence, $0<\alpha \leq 1$-cf. Theorem 3.1 of Mittal and Ylvisaker (1975)) 
then there exists a separable standard stationary Gaussian process $Y_{n}(t), n \in \mathbb{N}$, with correlation function

$$
\rho_{n, T(n)}(t)=\frac{r_{n}(t)-r_{n}(T(n))}{1-r_{n}(T(n))} \quad \text { for } t \leq T(n) .
$$

Let

$$
M_{T(n)}(Y)=\max _{0 \leq t \leq T(n)} Y_{n}(t) \quad \text { and } \quad M_{T(n)}(-Y)=\max _{0 \leq t \leq T(n)}-Y_{n}(t) .
$$

Lemma 3.6. Let $Y_{n}(t)$ be defined as above. Under the conditions of Theorem 2.2, for any $\varepsilon>0$,

$$
\lim _{n \rightarrow \infty} \mathrm{P}\left\{\left|M_{T(n)}(Y)-b_{T(n)}\right|>\varepsilon r_{n}^{1 / 2}(T(n))\right\}=0
$$

and

$$
\lim _{n \rightarrow \infty} \mathrm{P}\left\{\left|M_{T(n)}(-Y)-b_{T(n)}\right|>\varepsilon r_{n}^{1 / 2}(T(n))\right\}=0
$$

are valid.

Proof. Since the proofs are similar, we only give the proof of (3.16). By the assumptions,

$$
\rho_{n, T(n)}(t)=\frac{r_{n}(t)-r_{n}(T(n))}{1-r_{n}(T(n))}=1-c_{n}(T(n))|t|^{\alpha}+\epsilon_{n}(t)|t|^{\alpha}
$$

as $t \rightarrow 0$, where $c_{n}(T(n))=c_{n} /\left(1-r_{n}(T(n))\right) \rightarrow 1$ as $n \rightarrow \infty$ and $\epsilon_{n}(t)=$ $\varepsilon_{n}(t) /\left(1-r_{n}(T(n))\right) \rightarrow 0$ as $t \rightarrow 0$, uniformly in $n$. Furthermore, for any $\varepsilon>0$, there exists $\gamma>0$ such that $\sup \left\{\left|\rho_{n, T(n)}(t)\right|, T \geq|t| \geq \varepsilon, n \in \mathbb{N}\right\}<\gamma<1$. Utilising the stationarity of $\left\{Y_{n}(t), 0 \leq t \leq T(n)\right\}$, Theorem 1.1(i), and the definition of $b_{T(n)}$, we have

$$
\begin{aligned}
& \mathrm{P}\left\{M_{T(n)}(Y)-b_{T(n)}>\varepsilon r_{n}^{1 / 2}(T(n))\right\} \\
& \leq([T(n)]+1) \mathrm{P}\left\{\max _{0 \leq t \leq 1} Y_{n}(t)>\varepsilon r_{n}^{1 / 2}(T(n))+b_{T(n)}\right\} \\
& \leq C_{6}([T(n)]+1)\left(\varepsilon r_{n}^{1 / 2}(T(n))+b_{T(n)}\right)^{2 / \alpha-1} \exp \left(-\frac{1}{2}\left(r_{n}^{1 / 2}(T(n))+b_{T(n)}\right)^{2}\right) \\
& \leq C_{6}([T(n)]+1)(\log T(n))^{(2-\alpha) / 2 \alpha} \\
& \times \exp \left(-\frac{1}{2}\left(2 \log T(n)+\frac{2-\alpha}{\alpha} \log \log T(n)+2\left(r_{n}(T(n)) \log T(n)\right)^{1 / 2}\right)\right) \\
& \leq C_{6} \exp \left(-\left(r_{n}(T(n)) \log T(n)\right)^{1 / 2}\right) .
\end{aligned}
$$

Assumption (B1) and the fact that $\lim _{n \rightarrow \infty} r_{n}(T(n)) \log T(n)=\infty$ imply that

$$
\lim _{n \rightarrow \infty} \mathrm{P}\left\{M_{T(n)}(Y)-b_{T(n)}>\varepsilon r_{n}^{1 / 2}(T(n))\right\}=0 .
$$

Next, repeating the proof of Equation (3.9) of Mital and Ylvisaker (1975), we have

$$
\lim _{n \rightarrow \infty} \mathrm{P}\left\{M_{T(n)}(Y)-b_{T(n)}<-\varepsilon r_{n}^{1 / 2}(T(n))\right\}=0 ;
$$

hence (3.16) holds, and thus the claim follows. 
Proof of Theorem 2.2. Represent $X_{n}(t)$ as

$$
X_{n}(t)=\left(1-r_{n}(T(n))\right)^{1 / 2} Y_{n}(t)+r_{n}^{1 / 2}(T(n)) \mathcal{W},
$$

where $\mathcal{W}$ is a standard Gaussian random variable independent of the process $\left\{Y_{n}(t), t \geq 0\right\}$. Using Lemma 3.6 and setting $a(n):=\sqrt{\left(1-r_{n}(T(n))\right) / r_{n}(T(n))}$, we obtain

$$
\begin{aligned}
\mathrm{P}\left\{r_{n}^{-1 / 2}(T(n))\left(\sup _{t \in[0, T(n)]}\left|X_{n}(t)\right|-\left(1-r_{n}(T(n))\right)^{1 / 2} b_{T(n)}\right) \leq x\right\} \\
\quad=\mathrm{P}\left\{\sup _{t \in[0, T(n)]}\left|X_{n}(t)\right| \leq r_{n}^{1 / 2}(T(n))\left[a(n) b_{T(n)}+x\right]\right\} \\
\quad=\mathrm{P}\left\{-x \leq a(n)\left(Y_{n}(t)+b_{T(n)}\right)+\mathcal{W}, a(n)\left(Y_{n}(t)-b_{T(n)}\right)+\mathcal{W} \leq x, t \in[0, T(n)]\right\} \\
\quad=\mathrm{P}\left\{a(n)\left(-Y_{n}(t)-b_{T(n)}\right)-\mathcal{W} \leq x, a(n)\left(Y_{n}(t)-b_{T(n)}\right)+\mathcal{W} \leq x, t \in[0, T(n)]\right\} \\
\quad=\mathrm{P}\left\{a(n)\left(M_{T(n)}(-Y)-b_{T(n)}\right)-\mathcal{W} \leq x, a(n)\left(M_{T(n)}(Y)-b_{T(n)}\right)+\mathcal{W} \leq x\right\} \\
\quad \rightarrow \mathrm{P}\{-\mathcal{W} \leq x, \mathbb{W} \leq x\} \quad \text { as } n \rightarrow \infty,
\end{aligned}
$$

and, hence, the claim follows.

\section{Acknowledgements}

We would like to thank the referees and the Editor for their comments and suggestions which greatly improved the manuscript. Z. Tan has been supported by the National Science Foundation of China under grant 11071182, E. Hashorva has been supported by the Swiss National Science Foundation under grant 200021-1401633/1, and Z. Peng has been supported by the National Natural Science Foundation of China under grant 11171275.

\section{References}

AdLer, R. J. (1990). An Introduction to Continuity, Extrema, and Related Topics for General Gaussian Processes (Inst. Math. Statist. Lecture Notes Monogr. Ser. 12). Institute of Mathematical Statistics, Hayward, CA.

Albin, J. M. P. And Chol, H. (2010). A new proof of an old result by Pickands. Electron. Commun. Prob. 15, $339-345$.

Berman, S. M. (1992). Sojourns and Extremes of Stochastic Processes. Wadsworth \& Brooks/Cole, Pacific Grove, CA.

DȩBicki, K. And Kisowski, P. (2009). A note on upper estimates for Pickands constants. Statist. Prob. Lett. 78, 2046-2051.

Dieker, A. B. (2005). Extremes of Gaussian processes over an infinite horizon. Stoch. Process. Appl. 115, $207-248$.

Durrett, R. (2004). Probability Theory and Examples. Duxbury Press, Boston.

Ho, H.-C. AND McCormick, W. P. (1999). Asymptotic distribution of sum and maximum for Gaussian processes. J. Appl. Prob. 36, 1031-1044.

Hüsler, J. (1999). Extremes of Gaussian processes, on results of Piterbarg and Seleznjev. Statist. Prob. Lett. 44, 251-258.

HÜsler, J., Piterbarg, V. and Seleznjev, O. (2003). On convergence of the uniform norms for Gaussian processes and linear approximation problems. Ann. Appl. Prob. 13, 1615-1653.

LeAdbetter, M. R., Lindgren, G. And Rootzén, H. (1983). Extremes and Related Properties of Random Sequences and Processes. Springer, New York.

Mittal, Y. AND Ylvisaker, D. (1975). Limit distributions for the maximum of stationary Gaussian processes. Stoch. Process. Appl. 3, 1-18.

PICKANDS, J., III. (1969). Asymptotic properties of the maximum in a stationary Gaussian process. Trans. Amer. Math. Soc. 145, 75-86.

Piterbarg, V. I. (1972). On the paper by J. Pickands 'Upcrossing probabilities for stationary Gaussian processes'. Vestnik Moscow. Univ. Ser. I Mat. Meh. 27, 25-30 (in Russian). English translation: Moscow Univ. Math. Bull.

Piterbarg, V. I. (1996). Asymptotic Methods in the Theory of Gaussian Processes and Fields. American Mathematical Society, Providence, RI. 
SeleznjeV, O. V. (1991). Limit theorems for maxima and crossings of a sequence of Gaussian processes and approximation of random processes. J. Appl. Prob. 28, 17-32.

SELEZnJev, O. (1996). Large deviations in the piecewise linear approximation of Gaussian processes with stationary increments. Adv. Appl. Prob. 28, 481-499.

SELEZnJev, O. (2006). Asymptotic behavior of mean uniform norms for sequences of Gaussian processes and fields. Extremes 8, 161-169.

SHAO, Q. M. (1996). Bounds and estimators of a basic constant in extreme value theory of Gaussian processes. Statistica Sinica 6, 245-257.

Stamatovic, B. And Stamatovic, S. (2010). Cox limit theorem for large excursions of a norm of Gaussian vector process. Statist. Prob. Lett. 80, 1479-1485. 